number of cysts on the second scan. Kidney volume decreased slightly more in patients who received sirolimus than in those who received tacrolimus. Liver sections from two patients exhibited intense cytoplasmic staining of activated mTOR in the cyst-lining epithelium; staining was minimal in sections obtained from two controls without ADPKD and from noncystic regions of PLD tissues.

The authors conclude that sirolimus might be a therapeutic option for patients with PLD, as liver enlargement is a major source of morbidity and mortality in this condition.

Original article Qian Q et al. (2008) Sirolimus reduces polycystic liver volume in ADPKD patients. J Am Soc Nephrol 19: 631-638

\section{Patients with nephrotic syndrome have a high absolute risk of thromboembolic events}

Data from a number of studies have indicated that patients with nephrotic syndrome (NS) are at an increased risk of thromboembolic events, but the absolute risks of venous thromboembolism (VTE) or arterial thromboembolism (ATE) in this patient population are unknown. To address this lack of information, Mahmoodi et al. assessed the incidences of ATE and VTE in a cohort of 298 patients with NS.

The mean age of the study population was 42 years and $59 \%$ of the cohort were male. During a mean follow-up of 10 years, 29 patients had at least one episode of VTE and 43 had at least one episode of ATE. The resulting annual incidence rates for VTE and ATE were $1.02 \%$ and $1.48 \%$, respectively. However, the incidences of VTE and ATE within 6 months of a diagnosis of NS were considerably higher than these data might indicate-the annual incidences of VTE and ATE on the basis of data obtained in the 6 months following NS diagnosis were $9.85 \%$ and $5.52 \%$, respectively. The ratio of proteinuria to serum albumin (a measure of the severity of NS) was significantly associated with the incidence of VTE (hazard ratio 5.6, 95\% Cl 1.2-26.2; $P=0.03$ ), but neither either parameter alone nor their ratio was predictive of ATE. The incidence of ATE was significantly associated with age, sex, hypertension, diabetes mellitus, smoking and estimated glomerular filtration rate $(P \leq 0.02$ for all).
The authors conclude that patients with NS have high absolute risks of VTE and ATE, particularly in the first 6 months after diagnosis.

Original article Mahmoodi BK et al. (2008) High absolute risks and predictors of venous and arterial thromboembolic events in patients with nephrotic syndrome: results from a large retrospective cohort study. Circulation 117: 224-230

\section{Simplifying nutritional screening in patients on hemodialysis}

Malnutrition is a common complication in patients on hemodialysis. The tools that have been validated to screen for risk of malnutrition in this setting-including the MalnutritionInflammation Score (MIS)-are complex, subjective assessments that must be carried out by dieticians who have received appropriate training. To identify an easier and more reliable means of pinpointing hemodialysis patients who are at risk of malnutrition, Yamada et al. compared five simplified nutritional screening tools, with the MIS used as a reference standard.

The screening tools tested were the Mini Nutritional Assessment-Short Form, the Nutrition Risk Score, the Malnutrition Universal Screening Tool, the Malnutrition Screening Tool, and the Geriatric Nutritional Risk Index (GNRI). A total of 422 hemodialysis patients (mean age 63.8 years) were recruited from two Japanese centers; progressive malnutrition was an exclusion criterion. The GNRI was found to have the greatest capacity to detect malnutrition (defined as an MIS $\geq 6$ ), because it had the greatest area under the receiver operating characteristic curve (0.84). This index can be calculated from a simple equation that requires only three variables (serum albumin, height, and body weight); no subjective assessment is involved. A GNRI score below 91.2 had a sensitivity of 0.730 and a specificity of 0.819 for predicting malnutrition; the accuracy, positive predictive value and negative predictive value were $0.787,0.717$ and 0.787 , respectively.

The authors conclude that, of the tools studied, the GNRI is the simplest and most accurate means of identifying malnutrition in patients receiving maintenance hemodialysis.

Original article Yamada K et al. (2008) Simplified

nutritional screening tools for patients on maintenance hemodialysis. Am J Clin Nutr 87: 106-113 